\title{
1 Nonadaptive Radiation: Pervasive diet specialization by drift in
} 2 scale insects?

3

4

5 Nate B Hardy ${ }^{1}$, Daniel A Peterson ${ }^{2}$, Benjamin B Normark ${ }^{2}$

6

$7 \quad{ }^{1}$ Department of Entomology and Plant Pathology, 301 Funchess Hall, Auburn University,

8 Auburn, Alabama 36849.

$9{ }^{2}$ Department of Biology and Graduate Program in Organismic \& Evolutionary Biology,

10 University of Massachusetts, Amherst, Massachusetts 01003.

12 Email: NBH (corresponding author): n8@auburn.edu, DAP: dapeters@cns.umass.edu, BBN:

13 bnormark@bio.umass.edu

15 Running head: Diet breadth drift in plant-feeding insects.

16 Keywords: insect-plant interactions, host range, niche breadth, diversification

17 Data archive: http://dx.doi.org/10.5061/dryad.925cb 


\section{Abstract}

20 At least half of metazoan species are herbivorous insects. Why are they so diverse? Most

21 herbivorous insects feed on few plant species, and adaptive host specialization is often invoked

22 to explain their diversification. Nevertheless, it is possible that the narrow host ranges of many

23 herbivorous insects are non-adaptive. Here, we test predictions of this hypothesis with

24 comparative phylogenetic analyses of scale insects, a group for which there appears to be few

25 host-use tradeoffs that would select against polyphagy, and for which passive wind-dispersal

26 should make host specificity costly. We infer a strong positive relationship between host range

27 and diversification rate, and a marked asymmetry in cladogenetic changes in diet breadth. These

28 results are consonant with a system of pervasive non-adaptive host specialization in which small,

29 drift- and extinction-prone populations are frequently isolated from persistent and polyphagous

30 source populations. They also contrast with the negative relationship between diet breadth and

31 taxonomic diversification that has been estimated in butterflies, a disparity which likely stems

32 from differences in the average costs and benefits of host specificity and generalism in scale

33 insects vs. butterflies. Our results indicate the potential for non-adaptive processes to be

34 important to diet-breadth evolution and taxonomic diversification across herbivorous insects.

\section{$37 \quad$ Keywords}

38 Insect-plant interactions, speciation, extinction, comparative analysis 


\section{Introduction}

42 Species richness is spread unevenly across the branches of the Tree of Life. What causes this

43 unevenness? One factor might be what a species eats. Herbivory has evolved repeatedly among

44 insects, and herbivorous insects tend to be more species-rich than their non-herbivorous relatives

45 (Mitter et al. 1988; Wiens et al. 2015; but see Rainford and Mayhew 2015). In fact, herbivorous

46 insects are more species-rich than any other group of metazoans (Futuyma and Agrawal 2009).

47 Understanding what drives species diversification in herbivorous insects is tantamount to

48 understanding most of species diversification. Host-plant associations are thought to be key

49 (Ehrlich and Raven 1964; Futuyma and Moreno 1988; Futuyma and Agrawal 2009).

51 Herbivorous insects vary conspicuously in their diet breadth. Most are host-plant specialists, but

52 frequency distributions of host ranges are right-skewed (e.g., Fig 1), and herbivorous insect

53 communities invariably include a minority of broad-diet (polyphagous) species (Forister et al.

54 2015). For the most part, we have assumed that host specialization is adaptive for herbivorous

55 insects and we have incorporated this assumption into our explanations of herbivorous insect

56 diversification (Ehrlich and Raven 1964). The Oscillation Hypothesis (Janz et al. 2006) is a good

57 example. It explains herbivorous insect diversification as adaptive radiation (Yoder et al. 2010):

58 an ecological opportunity decreases competition, relaxes stabilizing selection on host use, and

59 causes the host range of a herbivorous insect species to expand. But host-range expansion is only

60 temporary, because as competition rebounds, host-use trade-offs select against a broad diet and

61 drive speciation through host specialization. Note that, in adaptive models, among-host

62 performance trade-offs are essential; without them, there is no cost for polyphagy. But evidence 
63 for these trade-offs has been hard to find. In fact, most studies have found instead that within

64 herbivorous insect populations “general vigor” genotypes outperform others across all viable

65 hosts (e.g., Forister et al. 2007; Agosta and Klemens 2009; Gompert et al. 2015). Host-use trade-

66 offs are also called into question by the results of comparative phylogenetic analyses: in

67 butterflies, host-use trade-offs do not select strongly enough against generalists so as to make

68 broad diets evolutionarily ephemeral (Hardy and Otto 2014; Hamm and Fordyce 2015), and in

69 armored scale insects host-use trade-offs do not constrain the phylogenetic history of host use

70 (Peterson et al. 2015). This raises the possibility that much of the host specificity observed in

71 herbivorous insects is not adaptive.

72

73 Instead, host specificity may evolve by genetic drift (Gompert et al. 2015). Small populations

74 may become geographically cut off from polyphagous source populations. In these isolated

75 groups, selection for adaptive polyphagy may not be strong enough to overcome the power of

76 genetic drift, or selection for polyphagy may be relaxed if ancestral hosts are absent in restricted

77 geographic ranges. We refer to this possibility as the specialization-by-drift hypothesis.

78 Regardless of how specialization comes about, it should increase the likelihood of extinction

79 (Simpson 1953; Jablonski 1986). And if it evolves through genetic drift, it may be especially

80 likely to reduce the potential for subsequent adaptive evolution, such as host switching or host

81 range expansion (Jaenike 1990). Hence, the specialists produced by genetic drift can be thought

82 of as evolutionary dead-ends. The idea of specialists as dead-ends is an old and conventional one

83 in evolutionary biology (Cope 1896; Huxley 1942; Simpson 1953; Day et al. 2016);

84 conventionally, specialists are thought to have a short-term advantage and long-term

85 disadvantage. In the specialization-by-drift model they enjoy no advantage at all. 
87 In short, the standard adaptive-specialization hypothesis views generalists as unstable and

88 ephemeral, rather like radioactive isotopes that rapidly decay, whereas the specialization-by-drift

89 hypothesis views generalists as stable and persistent, like a kind of phylogenetic meristem.

90 Remarkably, although the reasons differ, the standard adaptive-specialization hypothesis and the

91 specialization-by-drift hypothesis make many of the same predictions about the evolution of host

92 range and its effects on species diversification. Both hypotheses predict that 1) host range

93 evolution will be tightly linked to speciation events, 2) host ranges will shrink more than expand,

94 and 3) generalists will speciate more rapidly than specialists.

96 To reiterate, in the standard version of the adaptive-specialization hypothesis, a rare ecological

97 opportunity causes the host range of a herbivorous insect species to expand. But before long,

98 escalating competition renders the performance trade-offs that are inherent to polyphagy

99 untenable, and the generalist rapidly decays into an array of specialist descendants. The adaptive-

100 specialization hypothesis doesn't rule out the possibility that specialists can speciate. But it does

101 imply that speciation rates are much faster in generalists, and that speciation-via-specialization is

102 the norm. On the other hand, in specialization-by-drift, generalist species stay generalist. But

103 because a generalist species is so widespread and flexible, there is a good chance that small

104 populations will become isolated somewhere along or beyond the edge of its geographic range. If

105 such a population is isolated for long enough, the evolution of reproductive isolation is likely,

106 and the evolution of host specificity may result from genetic drift and relaxed selection for

107 polyphagy. If environments change for these specialist species, and select for broader host

108 ranges, this selection will be inefficient, as it will be opposed by strong genetic drift, and some of

109 the genetic underpinnings of phenotypic plasticity will have decayed. Host specificity is a dead

110 end. Here we are contrasting one standard adaptive model with one plausible nonadaptive model; 
111 a range of other adaptive and non-adaptive hypotheses about host range are of course possible --

112 see Discussion.

113

114 The specialization-by-drift and standard adaptive-specialization hypotheses do make some

115 distinct and testable predictions about the phylogenetic evolution of host range. Specialization-

116 by-drift predicts that broad host ranges will persist over evolutionary time scales; that is, host

117 range will have a strong phylogenetic signal. By contrast, the adaptive-specialization hypothesis

118 predicts that broad host ranges will be ephemeral, as host-use tradeoffs select against generalist

119 phenotypes and hosts are rapidly apportioned among specialist daughter lineages. Specialization-

120 by-drift also predicts that most cladogenetic change in host range will be asymmetrical. One of

121 the daughter lineages produced by speciation of a polyphagous ancestor will be a host-specialist.

122 The other will retain the host range of the ancestral population. On the other hand, standard

123 adaptive-specialization predicts more symmetry in cladogenetic range reductions.

125 Comparative phylogenetic analyses of butterflies do not support the standard adaptive-

126 specialization hypothesis: species diversification rates appear higher in specialists, host range

127 size infrequently changes during speciation events, and when it does, expansion is as likely as

128 contraction (Hardy and Otto 2014). Furthermore, as noted previously, polyphagy is

129 phylogenetically conservative, at least at the level of butterfly genera (Hamm and Fordyce 2015;

130 but see Nylin et al. 2014). Here, we estimate how host range affects species diversification in

131 scale insects, a group for which we expect specialization-by-drift to be especially likely. Our

132 expectation is based on three considerations: 1) Comparative phylogenetic analyses indicate a

133 lack of evolutionary tradeoffs in host use that would select against generalist phenotypes in scale

134 insects (Peterson et al. 2015), that is, polyphagy is cheap. 2) Scale insects have sessile adult 
135 females and disperse passively, by wind, as delicate first instar nymphs that can not survive for

136 long away from a host. Thus, in scale insects, host specialization may be especially costly, due

137 to high mortality during dispersal (Dixon et al. 1987). 3) Polyphagous scale insect species can

138 have exceptionally broad host ranges, geographic ranges, and population sizes (García Morales

139 et al. 2016; Ross et al. 2013). These are species that would seem to have an exceptionally low

140 probability of going extinct, and a high probability of giving rise to isolated offshoots.

141

142 Methods

143 Statistical tests were performed in the R software environment (R core development team 2015)

144 on the comparative phylogenetic dataset of Hardy et al. (2015). The data are in the Dryad archive

145 (http://dx.doi.org/10.5061/dryad.925cb). They consist of a time-calibrated, multi-locus estimate

146 of phylogeny for 472 scale insect species, along with host range estimates for 426 of the species

147 in the phylogeny. Hardy et al. (2015) quantified host ranges as counts of host plant families and

148 as phylogenetic diversities (PD) of host genera.

150 Here, we estimated the effect of host range on taxonomic diversification rates by fitting

151 Quantitative-state Speciation and Extinction (QuaSSE) models (FitzJohn 2010). Specifically, we

152 compared a model in which speciation rate was a linear function of host range to a model in

153 which speciation rate was constant and independent of host range. In both models, constant

154 extinction rates were estimated. SSE model comparisons based on empirical phylogenies are

155 prone to elevated Type I error rates (Madisson and FitzJohn 2014; Rabosky and Goldberg 2015).

156 To address this issue, we used a simulation approach to adjust significance thresholds, following

157 the recommendation of Rabosky and Goldberg (2015). Specifically, we constructed a set of 100 
158 null datasets by simulating the evolution of a diversification-rate-neutral trait over the scale

159 insect phylogeny, using a Brownian Motion model with the value of the $\sigma^{2}$ parameter estimated

160 from the empirical data. Then, for each simulated dataset, we calculated the difference between

161 AIC scores ( $\delta$-AIC) of a constant- and linear-speciation-rate QuaSSE model. Lastly, we

162 determined the significance of the empirical $\delta$-AIC through comparison to the null distribution of

163 simulated $\delta$-AICs.

164

165 To assess the frequency and symmetry of cladogenetic changes in host range, we transformed the

166 host range data to a binary trait by coding species that feed on hosts in one plant family as

167 monophagous, and species that feed on two or more host plant families as polyphagous. Then we

168 compared the fit of four Binary State Speciation and Extinction node-enhanced state shift

169 (BiSSE-ness: Magnuson-Ford and Otto 2012) models: 1) a model in which there was only

170 anagenetic evolution of host range size (this has six free parameters and is equivalent to the

171 standard BiSSE model), 2) a model in which there is only cladogenetic evolution of host range,

172 and the rates and symmetry of host range evolution depend on range size (this has eight free

173 parameters), 3) a cladogenetic model in which the proportion of asymmetrical host range

174 evolution in monophagous ancestors (i.e., the p0a paramter) was set to 0.5 (seven free

175 parameters), and 4) a cladogenetic model in which in which the proportion of asymmetrical host

176 range evolution in polyphagous ancestors $(p 1 a)$ was set to 0.5. Full model specifications are

177 available in the R scripts in Supplementary Materials. The significance of model comparisons

178 was evaluated using simulation, as for the QuaSSE analyses, except with different models of

179 neutral trait evolution. For the comparison between the anagenetic and full cladogenetic models,

180 we used a mk2 model of discrete trait evolution to simulate neutral trait histories. For the 
181 comparisons between the full and constrained cladogenetic models, we evolved traits under an

182 mk2 model over a version of the scale insect phylogeny in which all branch length were set to 1.

183 Transforming branch lengths in this way approximates a history of cladogenetic (i.e., punctuated)

184 evolution (Pagel 1994). We fit all SSE models using maximum likelihood optimization in the

185 diversitree package (FitzJohn 2012). We also performed a Bayesian search to get an additional

186 sense of the error of the linear-speciation-rate QuaSSE model coefficient estimates. We ran an

187 MCMC analysis for 1000 steps, using the ML parameter estimates as starting values, and an

188 exponential prior with rate 10 . The first 100 steps were discarded as burn-in. We used Geweke's

189 convergence diagnostic to confirm that the remaining 900 samples were from the stationary

190 distribution (Geweke 1992; Plummer et al. 2006).

191

192 To visualize the evolution of host range over the scale insect phylogeny, we performed an

193 ancestral state reconstruction under the estimated parameters of a BiSSE model. Lastly, we

194 estimated the phylogenetic signal of host range using Abouheif's test (Abouheif 1999) and

195 orthonormal decomposition (Ollier et al. 2006), implemented in the R package adephylo

196 (Jombart et al. 2010).

\section{Results}

198 We found a positive relationship between host range and diversification rate, regardless of how

199 host range was measured, or what estimation procedure was used (Fig 1). The linear speciation

200 model fit the data better than the constant speciation model (host family counts $\delta$-AIC $=42.3$, p-

201 value $=0.02 ; \mathrm{PD} \delta$-AIC $=49.7$, p-value $<0.01$, and had a positive slope (Table S1).

202 Interestingly, in the PD parameterization, the speciation rate estimates for the most host specific

203 species were lower than the estimated extinction rate. We also found that the evolution of host 
204 range size is largely cladogenetic and strongly asymmetrical. The binary-state model with only

205 cladogenetic evolution of host range was strongly preferred to a model with only anagenetic host

206 range evolution $(\delta$-AIC $=14.8$, p-value $<0.01)$. Moreover, in the cladogenetic model, the

207 proportion of asymmetrical host range changes in speciation events involving polyphagous

208 ancestors was close to unity (Table S1). The full cladogenetic model was a significantly better fit

209 to the data than a model in which cladogenetic changes in polyphagous ancestors were

210 constrained to be symmetrical $(\delta$-AIC $=10.5$, p-value $<0.01)$. By constrast, constraining

211 monophagous ancestors to be involved in only symmetrical cladogenetic changes in host range

212 did not significantly reduce the likelihood of the model at the 0.5 level $(\delta$-AIC $=1.7$, $\mathrm{p}$-value $=$

213 0.07). Note that in the best-fitting binary-state model, the full cladogenetic model, speciation

214 rates are actually faster in specialist lineages, but so are extinction rates, and as a result, net

215 diversification rates are an order of magnitude higher in generalists (Table S1).

216

217 We recovered strong phylogenetic signal for host range (Abouheif's test, Dmax and SCE statistic

218 p-values each $<0.01$ ), although we should point out that those tests assume that host range

219 evolves under Brownian Motion and does not affect taxonomic diversification. The phylogenetic

220 conservatism of polyphagy is striking in the BiSSE reconstruction of ancestral host ranges (Fig

221 2.)

\section{Discussion}

223 In this study, we find support for two predictions of the standard adaptive-specialization

224 hypothesis: 1) a positive relationship between host range and species diversification rate, and 2)

225 a high proportion of cladogenetic host range reduction. This constitutes the first support for those

226 predictions, and contrasts with the case of butterflies, which display a negative effect of host 
227 range size on diversification rate, and a low proportion of cladogenetic host range evolution

228 (Hardy and Otto 2014). However, both of these predictions are also consistent with the

229 specialization-by-drift hypothesis, and for scale insects, we expect specialization-by-drift to be

230 more likely. Indeed, we recover two further patterns that are consistent with specialization-by-

231 drift but not with the standard adaptive-specialization hypothesis: 3) asymmetry of cladogenetic

232 host range reductions, with specialization occurring in only one of the pair of daughter species,

233 and 4) a strong phylogenetic signal for host range, with polyphagy persisting over evolutionary

234 time. Nevertheless, the phylogenetic patterns of host-use evolution do not tell us anything

235 directly about the short-term adaptiveness of host specificity in scale insects, and could be

236 explained by alternative hypotheses.

237

238

One such alternative is the serial-specialization hypothesis, first applied to parasitic tachinid flies

239 (Stireman 2005). The starting premise is that, depending on the circumstances, selection may

240 push for small host ranges or large ones. Examples of factors that could determine optimal host

241 ranges for herbivorous insects include the stability of plant communities (Jaenike 1990) and the

242 dispersal ability of the herbivores (Hardy et al. 2015). The serial-specialization hypothesis builds

243 on that premise with two additional assumptions. First, generalists are less likely to go extinct.

244 This is the flip side of the specialization as a dead-end hypothesis, and is supported by the fossil

245 record and comparative phylogenetics (e.g., Jablonski 1987, Hardy and Otto 2014). Second,

246 selection on local populations of a generalist species will often promote the evolution of host

247 specificity and reproductive isolation. The concept of an immortal generalist species that buds

248 off specialist daughter species is obviously something that serial-specialization shares with

249 specialization-by-drift. And this means that phylogentic patterns of host range evolution that we 
250

251

252

253

254

255

256

257

258

259

260

261

262

263

264

265

266

267

272 For other insects, especially insects with more efficient dispersal modes, host specialization by

273 drift may be less likely. For example, in butterflies, the vagility, longevity and complex sensory

estimated for scale insects are equally compatible with serial-specialization. How do we tell them apart?

Based on what else we know about scale insect biology, we think that specialization-by-drift is the more likely explanation. Specifically, in scale insects it looks like broad diets are cheap (Peterson et al. 2015), and narrow diets are expensive, owing to the mortality they are likely to cause during dispersal. But we cannot rule out the serial version of adaptive specialization; indeed, serial adaptive specialization may be highly plausible in some circumstances. The cost of host specificity in scale insects undoubtedly depends on the ecological and population genetic context (Hardy et al. 2015). For example, the costs of wind dispersal should be much lower in environments low plant species richness, or in which one plant species is extremely abundant. In fact, many specialist species of scale insects do maintain large populations on ecologically dominant hosts such as grasses and pines (García Morales et al. 2016). On the other hand, most specialist scale insect species occur in more diverse biomes and on less abundant hosts (García Morales et al. 2016). Which again points to more specialization by drift than by serial adaptation. But a glance at the phylogenetic distribution of host ranges in Figure 2 suggests that processes other than strict specialization by drift. Under the most extreme form of the specialization-bydrift hypothesis, one supreme generalist would be the progenitor of all other species, all of them specialists. In that case, barring some hard polytomies, the scale insect phylogeny would be completely unbalanced, and the generalist would be sister to its most recent offshoot (Stireman 2005). That's not what Figure 2 looks like.

(1)


274 systems of adult females may dramatically lower costs for host-plant specialization, and could

275 open the door to more adaptive specificity. On the other hand, the costs of polyphagy are as

276 unclear for butterflies as they are for scale insects, and that leaves the door open for non-adaptive

277 specificity. Butterfly life history may also serve to make specialization-by-drift harder to detect;

278 it may be more likely that specialization by drift is followed by adaptive niche transformation

279 and expansion, a process that would be consistent with the symmetrical evolutionary changes in

280 host range estimated by Hardy and Otto (2014). But the most likely candidates for specialization-

281 by-drift are other groups for which specificity is especially costly; that is, groups with low-

282 mobility adult females and haphazard dispersal such as root-feeding weevils (Curculionidae:

283 Entiminae), stick insects (Phasmatodea), and bagworm moths (Psychidae) (Normark and

284 Johnson 2011).

285

286 In our description of specialization-by-drift we assumed an allopatric mode for speciation.

287 Alternative non-adaptive modes of speciation, such as conflictual speciation (Crespi and Nosil,

288 2013), might also be relevant. This is especially true for scale insects, which engage in obligate

289 endosymbioses that are expected to increase the potential for genetic conflict (Brucker and

290 Bordenstein 2012). However, unless strong barriers to hybridization are initially present, genes

291 involved in conflicts between scale insects and their endosymbionts might actually homogenize

292 gene pools, and forestall speciation (Crespi and Nosil 2013). Which mean that genetic conflicts

293 probably play a more important role in crystallizing the genetic divergences which occurred in

294 allopatry, than in driving sympatric speciation.

295

296 Our results imply that, for most scale insects, ecological specificity is a dead-end. The evidence

297 is mixed for ecological specificity being a dead-end in other lineages (Jablonski 2008; Day et al. 
2016). This is not surprising, given the dissonance in the theory for how specificity should affect

299 macroevolution. On the one hand, we have the dead-end. On the other, the master of a trade. As Jablonski (2008) points out in his excellent review of species selection, most of the traits that we

301 expect to increase the risk of extinction should also increase the chance of speciation. Ecological 302 specificity falls into this category. Furthermore, if specialization increases competitive ability, it 303 might actually decrease the risk of extinction (Roberts and Hawkins 1999; Stireman 2005). The 304 net effect that it has on species diversification should depend on the relative costs of big niches 305 (e.g., inefficient metabolism) and small niches (e.g., smaller, patchier resources), which should 306 vary among species, depending on factors such as dispersal mode and environmental 307 heterogeneity.

309 Our results are contingent on the parameters that were included in the models of trait-dependent 310 diversification and on the assumptions made by those models. Host range per se cannot be the 311 most proximate factor affecting speciation and extinction dynamics. Rather, host range should 312 affect diversification by influencing population genetic parameters like population size and 313 structure, by playing a role in structuring the selective environment, and by affecting the 314 probability of colonizing new hosts and new places (Jahner et al. 2011; Slove and Janz 2011). In 315 this study we fail to reject the hypothesis that host specialization is caused by genetic drift in 316 scale insects. Further research is needed to test other aspects of that hypothesis, for example 317 comparative assessments of population size and structure in generalist and specialist species, and 318 experiments designed to assess the extent of drift, or the strength of selection at loci important 319 for host use and across the genome. 


\section{Conclusions}

321

Most herbivorous insect species are host-plant specialists. The idea that a specialist on its own

322 host should be able to outperform generalists is intuitively appealing, but it fails to account for

323 how host specificity may alter population genetic factors and the efficiency of natural selection.

324 It also fails to align with the ever-expanding body of research that indicates the superior

325 performance of generalists, and fails to predict the distribution of host range across herbivorous

326 insect phylogenies (e.g., Hardy \& Otto 2014, Peterson et al. 2015). Here we find patterns of host

327 range evolution that are consistent with a model of specialization by drift. Population genetic

328 processes other than natural selection should be more fully and more routinely incorporated into

329 ideas about the species diversification of the most species-rich metazoans.

330

331

332

334

335

336

\section{References}

Abouheif, E. 1999. A method for testing the assumption of phylogenetic independence in comparative data. Evol. Ecol. Res. 1:895-909.

Agosta, S. J., and J. A. Klemens. 2009. Resource specialization in a phytophagous insect: no evidence for genetically based performance trade-offs across hosts in the field or laboratory. J.

Evol. Biol. 22:907-912. 
Brucker, R.M. and S. R. Bordenstein. 2012. Speciation by symbiosis. Trends Ecol. Evol. 27: 443-451.

Cope, E. D. 1896. The primary factors of organic evolution. Open Court Publishing CO., Chicago.

Crespi, B. and P. Nosil. 2013. Conflictual speciation: species formation via genomic conflict. Trends Ecol. Evol. 28: 48-57.

Day, E. H., X. Hua and L. Bromham. 2016. Is specialization an evolutionary dead end? Testing for differences in speciation, extinction and trait transition rates across diverse phylogenies of specialista and generalists. J. Evol. Bio. DOI: 10.1111/jeb.12867

Dixon, A. F. G., P. Kindlmann, J. Lepš, and J. Holman. 1987. Why there are so few species of aphids, especially in the tropics. Am. Nat. 129:580-592.

Ehrlich, P. R., and P. H. Raven. 1964. Butterflies and plants: a study in coevolution. Evolution 586-608.

FitzJohn, R. G. 2012. Diversitree: comparative phylogenetic analyses of diversification in R. Methods Ecol. Evol. 3:1084-1092.

FitzJohn, R. G. 2010. Quantitative traits and diversification. Syst. Biol. 59:619-633.

Forister, M. L., A. G. Ehmer and D. J. Futuyma. 2007. The genetic architecture of a niche: variation and covariation in host use traits in the Colorado potato beetle. J. Evol. Bio. 20:985996. 
Forister, M. L., V. Novotny., A. K. Panorska, L. Baje, Y. Basset, P. T. Butterill, L. Cizek, P. D. Coley, F. Dem, I. R. Diniz and P. Drozd. 2015. The global distribution of diet breadth in insect herbivores. Proc. Natl. Acad. Sci. 112:442-447.

Futuyma, D. J., and A. A. Agrawal. 2009. Macroevolution and the biological diversity of plants and herbivores. Proc. Natl. Acad. Sci. 106:18054-18061.

Futuyma, D. J., and G. Moreno. 1988. The evolution of ecological specialization. Annu. Rev. Ecol. Syst. 19:207-233.

GarcÍa Morales, M., B. D. Denno, D. R. Miller, G. L. Miller, Y. Ben-Dov and N. B. Hardy. 2016. ScaleNet: a literature-based model of scale insect biology and systematics. Database 2016:bav118, doi: 10.1093/database/bav118

Geweke, J. Evaluating the accuracy of sampling-based approaches to calculating posterior moments. In Bayesian Statistics 4 (ed JM Bernado, JO Berger, AP Dawid and AFM Smith). Clarendon Press, Oxford, UK.

Gompert, Z., J. P. Jahner, C. F. Scholl, J. S. Wilson, L. K. Lucas, V. Soria-Carrasco, J. A. Fordyce, C. C. Nice, C. A. Buerkle, and M. L. Forister. 2015. The evolution of novel host use is unlikely to be constrained by tradeoffs or a lack of genetic variation. Mol. Ecol. 24:2777-2793.

Hamm, C. A., and J. A. Fordyce. 2015. Patterns of host plant utilization and diversification in the brush-footed butterflies. Evolution 69:589-601.

Hardy, N. B. and L. G. Cook. 2010. Gall-induction in insects: evolutionary dead-end or speciation driver? BMC Evol. Bio. 10:257; DOI: 10.1186/1471-2148-10-257 
Hardy, N. B., and S. P. Otto. 2014. Specialization and generalization in the diversification of phytophagous insects: tests of the musical chairs and oscillation hypotheses. Proc. R. Soc. B Biol. Sci. 281:20132960.

Hardy, N. B., D. A. Peterson and B. B. Normark. 2015. Scale insect host ranges are broader in the tropics. Bio. Let. 11: 20150924; DOI: 10.1098/rsbl.2015.0924

Huxley, J. 1942. Evolution: the modern synthesis. George Allen and Unwin, London.

Jaenike, J. 1990. Host specialization in phytophagous insects. An. Rev. Ecol. Sys. 21: 243-273.

Jablonski, D. 1986. Background and mass extinctions: The alteration of macroevolutionary regimes. Science 231: 129-133.

Jablosnki, D. 2008. Species selection: Theory and Data. Annu. Rev. Ecol. Evol. Syst. 39: 501524.

Jahner, J. P., M. M. Bonilla, K. J. Badik, A. M. Shapiro and M. L. Forister 2011. Use of exotic hosts by Lepidoptera: widespread species colonize more novel hosts. Evolution 65: 2719-2724.

Janz, N., S. Nylin, and N. Wahlberg. 2006. Diversity begets diversity: host expansions and the diversification of plant-feeding insects. BMC Evol. Biol. 6:4.

Jombart, T., F. Balloux, and S. Dray. 2010. adephylo: new tools for investigating the phylogenetic signal in biological traits. Bioinformatics 26:1907-1909.

Kelley S. T., and B. D. Farrell. 1998. Is specialization a dead end? The phylogeny of host use in Dendroctonus bark beetles (Scolytidae). Evolution 52:1731-1743. 
Maddison, W. P., and R. G. FitzJohn. 2015. The Unsolved Challenge to Phylogenetic Correlation Tests for Categorical Characters. Syst. Biol. 64:127-136.

Magnuson-Ford, K., and S. P. Otto. 2012. Linking the investigations of character evolution and species diversification. Am. Nat. 180:225-245.

Mitter, C., B. Farrell, and B. Wiegmann. 1988. The Phylogenetic Study of Adaptive Zones: Has Phytophagy Promoted Insect Diversification? Am. Nat. 132:107-128.

Normark, B. B., and N. A. Johnson. 2011. Niche explosion. Genetica 139:551-564.

Nylin, S., J. Slove and N. Janz. 2014. Host plant utilization, host range oscillations and diversification in nymphalid butterflies: a phylogenetic investigation. Evolution 68:105-124.

Ollier, S., P. Couteron, and D. Chessel. 2006. Orthonormal Transform to Decompose the Variance of a Life-History Trait across a Phylogenetic Tree. Biometrics 62:471-477.

Pagel, M. 1994. Detecting correlated evolution on phylogenies: a general method for the comparative analysis of discrete characters. Proc. R. Soc. Lond. B. 255:37-45.

Peterson, D. A., N. B. Hardy, G. E. Morse, I. C. Stocks, A. Okusu, and B. B. Normark. 2015. Phylogenetic analysis reveals positive correlations between adaptations to diverse hosts in a group of pathogen-like herbivores. Evolution 69:2785-2792.

Plummer, M., N. Best, K. Cowles and K. Vines. 2006. CODA: Convergence diagnosis and output analysis for MCMC. R news 6:7-11.

Rabosky, D. L., and E. E. Goldberg. 2015. Model Inadequacy and Mistaken Inferences of TraitDependent Speciation. Syst. Biol. Syu131. 
Rainford, J.L. and Mayhew, P.J., 2015. Diet evolution and clade richness in Hexapoda: a

phylogenetic study of higher taxa. Am. Nat. 186:777-791.

Roberts, C. M. and J. P. Hawkins. 1999. Extinction risk in the sea. Trends Ecol. Evol. 14: 241246.

Simpson, G. 1953. The major features of evolution. Colombia Univ. Press, New York.

Slove, J., and N. Janz. 2011. The relationship between diet breadth and geographic range size in the butterfly subfamily Nymphalinae—a study of global scale. PLoS ONE 6:e16057.

Stireman, J. O. 2005. The evolution of generalization? Parasitoid flies and the perils of inferring host range evolution from phylogenies. J. Evol. Bio. 18: 325-336.

Wiens, J. J., Lapoint, R. T. and Whiteman, N.K. 2015. Herbivory increases diversification across insect clades. Nature communications 6:8370, DOI: 10.1038/ncomms9370.

Yoder, J.B., E. Clancey, S. Des Roches, J. M. Eastman, L. Gentry, W. Godsoe, T. J. Hagey, D.

Jochimsen, B. P. Oswald, J. Robertson and B. A. J. Sarver. 2010. Ecological opportunity and the origin of adaptive radiations. J. Evol. Bio. 23: 1581-1596. 
337 Figure 1. Speciation rate estimated as a function of host plant range (number of host families).

338 The number of host families used by each of the 426 scale insect species examined is shown as a

339 histogram. The solid black line shows the Bayesian estimate of the mean relationship between

340 host range and speciation under a QuaSSE model (speciation rate $=0.095+0.050 *$ host range).

341 The dashed gray lines show the 95\% High Posterior Density.

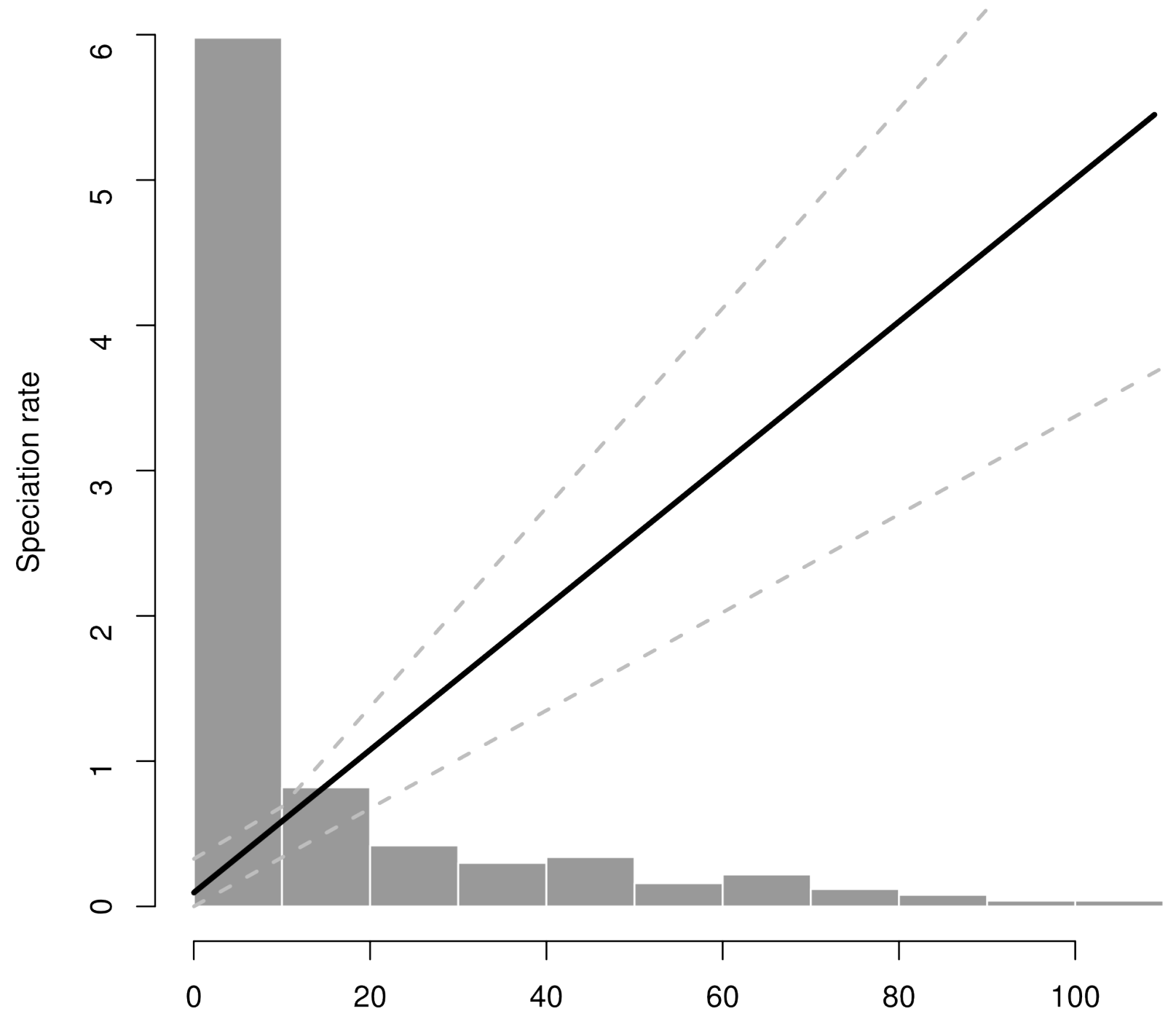

No. host families 
bioRxiv preprint doi: https://doi org/10 1101/064220; this version posted July 15 2016. The copyright holder for this preprint (which was not certified by peer review) is the author/funder, who has granted bioRxiv a license to display the preprint in perpetuity. It is made available under aCC-BY 4.0 International license.

345 Figure 2. Marginal ancestral state reconstructions under the estimated BiSSE model parameters.

346 Pie charts show proportional likelihoods at internodes. Gray: monophagy (one host plant family),

347 Green: polyphagy (multiple host plant families).

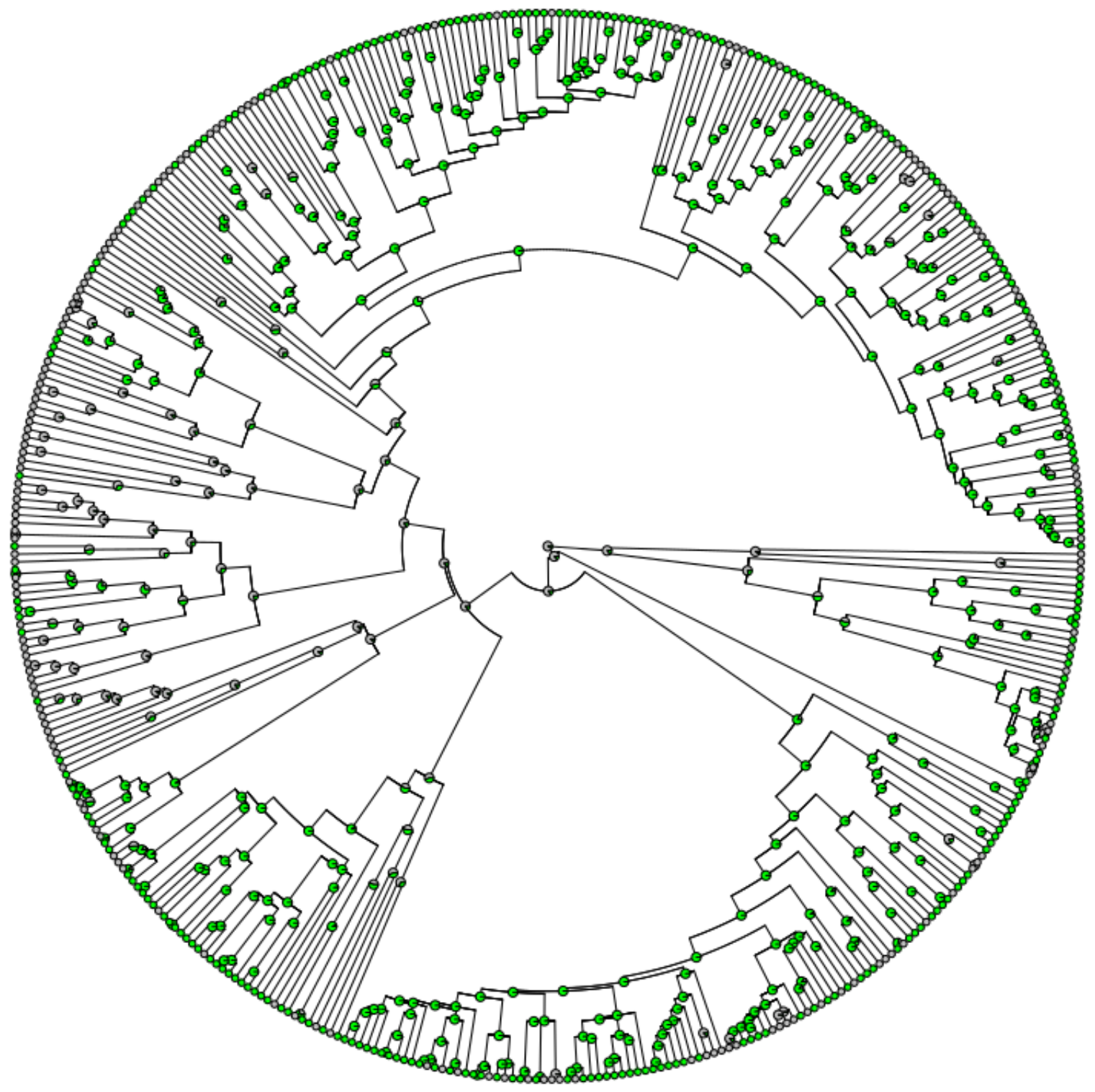

